\title{
Imaging Chromophores With Undetectable Fluorescence by Stimulated Emission Microscopy
}

\section{Citation}

Min, Wei, Sijia Lu, Shasha Chong, Rahul Roy, Gary R. Holtom, and Xiaoliang Sunney Xie. 2009. Imaging chromophores with undetectable fluorescence by stimulated emission microscopy. Nature: 461(7267): 1105-1109.

\section{Published Version}

doi:10.1038/nature08438

\section{Permanent link}

http://nrs.harvard.edu/urn-3:HUL.InstRepos:3693471

\section{Terms of Use}

This article was downloaded from Harvard University's DASH repository, and is made available under the terms and conditions applicable to Other Posted Material, as set forth at http:// nrs.harvard.edu/urn-3:HUL.InstRepos:dash.current.terms-of-use\#LAA

\section{Share Your Story}

The Harvard community has made this article openly available.

Please share how this access benefits you. Submit a story.

\section{Accessibility}




\section{Imaging Chromophores with Undetectable}

\section{Fluorescence by Stimulated Emission Microscopy}

Wei Min*, Sijia Lu*, Shasha Chong, Rahul Roy, Gary R. Holtom, X. Sunney Xie $\dagger$

Department of Chemistry and Chemical Biology, Harvard University, Cambridge, MA 02138 (USA)

* These authors contributed equally to this work

† To whom correspondence should be addressed. E-mail: xie@chemistry.harvard.edu (X.S.X.) 
Fluorescence, i.e., spontaneous emission, is generally more sensitive than absorption measurement, and is widely used in optical imaging ${ }^{1,2}$. However, many chromophores, such as hemoglobin and cytochromes, absorb but have undetectable fluorescence because the spontaneous emission is dominated by their fast nonradiative decay ${ }^{3}$ and hence overwhelmed by background signals. By allowing stimulated emission to effectively compete with the nonradiative decay, here we make the chromphores detectable and report a new contrast mechanism for optical microscopy. In a pump-probe experiment, upon photoexcitation by a pump pulse, the sample is stimulated down to the ground state by a time-delayed probe pulse, whose intensity is concurrently increased. We extract the miniscule intensity increase with shot-noise limited sensitivity by using a lock-in amplifier and intensity modulation of the pump beam at a high megahertz-frequency. The signal is generated only at the laser foci due to the nonlinear dependence on the input intensities, which offers intrinsic three-dimensional optical sectioning capability. In contrast, conventional one beam absorption measurement suffers from poor sensitivity, lack of 3D sectioning capability, and complication by sample scattering. We demonstrate a variety of applications of stimulated emission microscopy, such as visualizing chromoproteins, non-fluorescent variants of the green fluorescent protein, monitoring lacZ gene expression with a chromogenic reporter, mapping transdermal drug distributions without histological sectioning, and label-free microvascular imaging based on endogenous contrast of hemoglobin. In all these cases, sensitivity has been orders of magnitude higher than those of spontaneous emission or absorption contrast, allowing more reporters for molecular imaging. 
The phenomenon of stimulated emission was first described by Einstein in $1917^{4}$. An atom or molecule in its excited state can be stimulated down to the ground state by an incident light field, resulting in the creation of a new coherent photon identical to those in the original incident field. This process only occurs when the frequency of the incident field matches the transition energy. Stimulated emission was later used as a fundamental principle for light amplification in the laser. ${ }^{5}$. The depopulation aspect of stimulated emission has been used for population dumping from excited $\operatorname{states}^{6}$, super-resolution fluorescence (STED) microscopy ${ }^{7}$, and fluorescence lifetime imaging ${ }^{8}$. Here we utilize the light amplification aspect of stimulated emission as a contrast mechanism for highly sensitive imaging of chromophores with undetectable fluorescence.

Those chromophores have very short lived excited states with much faster nonradiative decay rates than their spontaneous emission rates. As a result, their feeble fluorescence is overwhelmed by backgrounds, such as stray light, solvent Raman scattering, and detector dark counts. Our solution to this problem is to conduct a dual beam experiment in order to interrogate the short lived excited state by stimulated emission, which can compete with the non-radiative decay under strong enough stimulating field strength (Fig. 1a). The resulting "amplification" of the stimulation beam can then be detected in the presence of the background signals.

Considering the optical excitation at frequency $\omega_{01}$ (Fig. 1a), the absorption cross section $\sigma_{a b s[0 \rightarrow 1]}$ is $\sim 10^{-16} \mathrm{~cm}^{2}$ for a single chromophore at room temperature ${ }^{2,9}$. As shown in Fig. 1b, under a tightly focused laser beam with a beam waist area of $S\left(\sim 10^{-9}\right.$ 
$\mathrm{cm}^{2}$ ), the integrated intensity attenuation of the excitation beam, $\Delta I_{E} / I_{E}$, is proportional to the ratio between $\sigma_{a b s[0 \rightarrow 1]}$ and $S$ :

$$
\Delta I_{E} / I_{E}=-N_{0} \cdot \sigma_{a b s[0 \rightarrow 1]} / S
$$

where $N_{0}$ is the number of molecules in the ground state. For a single chromophore, $\Delta I_{E} / I_{E}$ is on the order of $10^{-7}$. Such small attenuation cannot be detected by conventional absorption microscopy. We note that single molecule absorption was previously achieved in cryogenic temperatures using a frequency modulation method ${ }^{10}$, which is however difficult to implement at room temperature because of the broad molecular absorption spectrum. Moreover, absorption measurement is often complicated by scattering when imaging biological specimen. Instead of detecting direct absorption, here we detect the stimulated emission followed by the excitation of the molecule.

According to Einstein ${ }^{4}$, the molecular cross section $\sigma_{\text {sti.em. }}$ for stimulated emission is comparable to $\sigma_{a b s}$, because of microscopic reversibility. Unlike the absorption which results in attenuation, the stimulation beam will experience an intensity gain after interacting with the molecules

$$
\Delta I_{S} / I_{S}=+N_{2} \cdot \sigma_{\text {sti.em. }[2 \rightarrow 3]} / S
$$

where $N_{2}$ is the number of excited molecules transiently probed by the stimulation pulses (Fig. 1b). For a single chromophore, $\Delta I_{S} / I_{S}$ is also $\sim 10^{-7}$. Without special techniques, such a small signal would be again buried in the noise $(\sim 1 \%)$ of the stimulation beam. 
To overcome this noise problem and promote the advantage of performing stimulated emission, we implemented a high-frequency (> $1 \mathrm{MHz})$ phase-sensitive detection technique. In so doing, the laser intensity fluctuation, which occurs primarily at low frequency ( $\mathrm{kHz}$ to $\mathrm{DC})$, can be circumvented, as has been previously applied in other spectroscopic ${ }^{11}$ and recently stimulated Raman scattering (SRS) microscopy ${ }^{12}$ and two photon absorption microscopy ${ }^{13}$. In the scheme shown in Fig. $1 \mathrm{~b}$ and $\mathrm{c}$, the intensity of the excitation beam is modulated at $5 \mathrm{MHz}$, and this creates a modulation of the stimulated emission signal at the same frequency, because only when the excitation beam is present can the gain of the stimulation beam occur. Such an induced modulation signal can then be sensitively extracted by a lock-in amplifier referenced to this high frequency. In this way, the stimulated emission signal can be detected against the vanishing laser noise with a superior sensitivity. In contrast, it is difficult to implement such modulation scheme for high sensitivity one-beam absorption measurement ${ }^{11}$.

Specifically, we use a $\sim 200 \mathrm{fs}$ (FWHM) pulse train for excitation, and another $\sim 200 \mathrm{fs}$ pulse train for stimulation. The time delay between these two pulse trains is chosen to be $\sim 300 \mathrm{fs}$ (Fig. 1c), which is shorter than the excited state lifetime (sub-ps) of the chromophores. This delay also eliminates contributions from other instantaneous optical processes, such as two photon absorption ${ }^{13}$, cross phase modulation and SRS ${ }^{12}$.

We conduct the experiment under a non-saturating condition of the four-level system (Fig. 1a). Under this condition, $N_{2}$ in equation (2) originates from a linear excitation: $N_{2} \propto N_{0} \cdot I_{E} \cdot \sigma_{a b s[0 \rightarrow 1]} / S$. This relation, together with equation (2), indicates that the final signal $\Delta I_{S}$ is linearly dependent on both $I_{E}$ and $I_{S}$, 


$$
\Delta I_{S} \propto N_{0} \cdot I_{E} \cdot I_{S} \cdot\left(\sigma_{a b s[0 \rightarrow 1]} / S\right) \cdot\left(\sigma_{\text {sti.em. }[2 \rightarrow 3]} / S\right)
$$

The overall quadratic power dependence, as experimentally demonstrated (Fig.2a), would allow three-dimensional (3D) optical sectioning, as in many other multi-photon techniques ${ }^{14,15}$. Such dependence also confirms the non-saturation condition within the intensity range used. Moreover, it offers, in principle, a spatial resolution of twice as high (in spatial frequency) as in conventional fluorescence microscopy.

Fig. $2 \mathrm{~b}$ shows the stimulated emission signal as a function of the time-delay between the excitation and stimulation pulses. In pump-probe experiment, the signal could arise from ground state recovery, excited state absorption or stimulated emission. Ground state recovery is avoided here by the large Stokes shift of stimulation beam from molecular absorption band. Excited state absorption is negligible compared to stimulated emission based on the observed intensity increase of the stimulation beam, consistent with a study of fluorescence quenching in STED microscopy ${ }^{16}$. The initial rise in Fig. $2 b$ is due to vibrational relaxation from level 1 to level 2 (Fig. 1a), while the subsequent decay indicates the short excited state lifetime ( $\sim 0.6 \mathrm{ps})$ of level 2, which underlies the undetectable fluorescence. Such a short lifetime also reduces the probability of going into the triplet state, effectively protecting the molecule from photo-bleaching. The stimulated emission spectrum (Fig. 2c), recorded by tuning the wavelength of the stimulated beam, is also in agreement with the reported emission spectrum of crystal violet in glycerol ${ }^{17}$, where the high viscosity increases the fluorescence quantum yield.

Fig. $2 \mathrm{~d}$ shows that the stimulated emission signal depends linearly on analyte concentration, as predicted by equation (3). This allows straightforward quantitative 
analysis. The limit of detection is $\sim 60 \mathrm{nM}$ for crystal violet with $1 \mathrm{sec}$ integration time. This sensitivity effectively corresponds to a few $(<5)$ molecules in focus, which has surpassed the detection limit of recently reported for SRS microscopy ${ }^{12}$ by orders of magnitude because of the strong electronic transition. The ultimate sensitivity would be achieved under the condition of saturated excitation but near-saturated stimulation, and is fundamentally determined by the shot noise of the stimulation beam $\left(\Delta I_{S} / I_{S} \sim 10^{-7.5}\right.$ for $\sim 1 \mathrm{~mW}$ averaged over $1 \mathrm{sec})$.

As the first biological application, we imaged distributions of chromoproteins in live $E$. coli cells. Genetically encodable chromoproteins, such as $\mathrm{gtCP}^{18}$ and cjBlue ${ }^{19}$, are naturally existing variants of green fluorescent proteins ${ }^{20}$, and only absorb light but do not fluoresce. When the gene encoding for gtCP is expressed in E. coli cells, tetrameric gtCP can be clearly visualized to reside homogeneously inside cytoplasm by stimulated emission microscopy (Fig. 3 a\&b). Similarly, the distribution of another chromoprotein, cjBlue, can be imaged (Fig. 3 c\&d). Unlike gtCP which is expressed in most cells, cjBlue is expressed in a fraction of them, as we see from the corresponding transmission image. Therefore, stimulated emission microscopy opens the possibility of visualizing chromoproteins that are not accessible by fluorescence microscopy.

Next, we show the stimulated emission imaging in live E. coli cells of a nonfluorescent indigo reaction product commonly used to assay gene expression. $\beta$ galactosidase catalyzes the cleavage of the glycosidic linkage of X-gal, a popular chromogenic substrate, eventually forming an indigo product after oxidation ${ }^{21}$. The gene encoding $\beta$-galactosidase, lacZ, is a classic reporter gene. Traditionally, the indigo product has to accumulate in sufficient concentration for its blue color to be visible ${ }^{21}$. 
With stimulated emission, the accumulation of the indigo product can now be monitored in wild-type cells without induction of lacZ (Fig. 3 e-g). The inhomogeneous distribution of indigo product inside individual cells (Fig. 3 e\&f) is consistent with the fact that this product is insoluble and tends to form localized precipitates. In contrast, the corresponding transmission image (Fig. 3 g) shows no signs of any color. We note that an assay using a fluorogenic substrate has been recently developed ${ }^{22}$, but it requires a microfluidics to enclose individual cells because the hydrolysis product there is quickly pumped out by the cell. Hence, stimulated emission microscopy allows monitoring of a product presumed to report basal lacZ gene activity with superb sensitivity.

Monitoring transdermal delivery of non-fluorescent drug with intrinsic 3D optical sectioning is another application. In particular, we show mapping of a cationic thiazine dye toluidine blue $\mathrm{O}$ (TBO) at both the cellular and tissue levels. Having a selective affinity for cancer cells in vivo, TBO is a photosensitizer in photodynamic therapy ${ }^{23,24}$. Subcellular localization of TBO is crucial since it influences both the level and the kinetics of apoptosis induction. However, it is difficult to image the true TBO distribution, because its fluorescence is quenched when bound to tissue substrates and only the nonspecific stain residue in the tissue retains its native fluorescence ${ }^{24}$. Independent of the complication from fluorescence contrast, stimulated emission microscopy is suitable for this problem. The stimulated emission image of TBO inside the cancer cells after incubation shows its local accumulation (Supplementary Fig. 1). When topically applied to skin tissue, being hydrophilic and water soluble, TBO is enriched in the center of the protein phase of the polygonal stratum corneum cells rather than in the intercellular space which is in lipid phase (Fig. 4a). At a $20 \mu \mathrm{m}$ deeper depth, TBO displays a rich 
subcellular distribution in the cytoplasm of epidermis where cellular proliferation actively takes place (Fig. 4b). These imaging results support the hydrophilic delivery pathway as well as the recent hypothesis of TBO binding to cytoplasmic RNA to initiate apoptosis ${ }^{23}$.

Finally, we demonstrate label-free imaging of microvascular based on endogenous contrast from non-fluorescent hemoglobin. Structure and hemodynamics of blood vessels play a major role in many biomedical processes such as angiogenesis in tumors ${ }^{25}$ and cerebral oxygen delivery in brain ${ }^{26,27}$. However, established techniques such as MRI, CT, PET, ultrasound, confocal and two-photon fluorescence microscopy either lack the spatial resolution needed for resolve individual capillaries or require exogenous contrast agents. Here we perform ex vivo stimulated emission imaging of the well-developed vascular network from a nude mouse ear, by exciting the Soret band of hemoglobin through efficient two-photon absorption ${ }^{28}$ and subsequently stimulating the emission from its $Q$ band which has longer excited state lifetime than Soret band ${ }^{29}$. As shown in Fig. 4c, blood vessels (in red) branch and loop around sebaceous glands (in green). In addition, single red blood cells can be clearly seen to reside within individual capillaries $(\sim 5 \mu \mathrm{m}$ in diameter). Two photon absorption has been recently developed to image hemoglobin ${ }^{13}$. We note that stimulated emission is more sensitive because it involves direct one-photon transition. Furthermore, our new technique offers the prospect of 3D mapping of blood oxygenation level for addressing broad physiological and pathological problems ${ }^{25-27}$.

We note that the complexity and cost of the instrument can be much reduced as fiber laser sources are adapted. Stimulated emission microscopy allows imaging of nonfluorescent chromophores with 3D optical sectioning and high sensitivity, and extends the repertoire of reporters for biological imaging beyond fluorophores. 


\section{Figure Captions}

Fig. 1. Principle of stimulated emission microscopy. a, Energy diagram of spontaneous emission, non-radiative decay and stimulated emission processes for a typical four-level energy system. Non-radiative decay dominates spontaneous emission for chromophores with undetectable fluorescence. However, when the stimulation field is designed to have the correct energy and timing, the stimulated emission can compete with non-radiative decay and become the dominating decay pathway. b, Stimulated emission microscopy. The incident excitation and delayed stimulation pulse trains are spatially overlapped and focused onto the common focal spot in the sample. A modulator switches the intensity of the excitation beam on-and-off at $5 \mathrm{MHz}$. The spectrally filtered stimulation beam is detected by a large-area photodiode (PD), and demodulated by a lock-in amplifier to create the image contrast while raster scanning the collinear exciting and stimulating beams. The relative energy gain or loss of the stimulation and excitation beam, respectively, for a single chromophore at the laser focus (area of $S$ ), is given by the

equations (inset). c, Principle of high-frequency modulation. As the intensity of the excitation beam is modulated on-and-off in time, the corresponding stimulated emission signal is also modulated at the same frequency. The stimulation pulses is made to lag behind the excitation pulses by an appropriate amount $\Delta t$. 
Fig. 2 Characterizations of stimulated emission microscopy. a, Stimulated emission signal from $30 \mu \mathrm{M}$ crystal violet/water solution is proportional to the product of excitation beam power, $I_{\mathrm{E}}$, and stimulation beam power, $I_{\mathrm{S}}$ b, Stimulated emission signal as a function of time delay between the excitation $(590 \mathrm{~nm})$ and the stimulation $(660 \mathrm{~nm})$ pulses. The decay of the signal $(\sim 0.6 \mathrm{ps})$ reflects the short excited state lifetime. The FWHM of the pulses is $\sim 200 \mathrm{fs}$. c, Measured stimulated emission spectrum is in agreement with the reported fluorescence spectrum of crystal violet in glycerol solution ${ }^{16}$. $590 \mathrm{~nm}$ excitation is fixed while the stimulation wavelength is scanned by tuning the other OPO wavelength. A time delay of $0.3 \mathrm{ps}$ is used. Inset is the absorption spectrum of crystal violet in water. d, Stimulated emission signal scales linearly with crystal violet concentration in aqueous solution. Continuous flow of the sample is used to replenish molecules. $590 \mathrm{~nm}$ and $660 \mathrm{~nm}$ for excitation and stimulation beam, respectively. $0.3 \mathrm{ps}$ time delay is used. Error bars show 1 s.d. of the signals from a $30 \mathrm{sec}$ recording. The detection limit was determined to be $60 \mathrm{nM}$ with a signal-to-noise ratio of 1:1 (Supplementary Fig. 3). The excitation and stimulation beam intensities at the focus are $0.2 \mathrm{~mW}\left(\sim 0.14 \mathrm{MW} / \mathrm{cm}^{2}\right)$ and $1 \mathrm{~mW}\left(\sim 0.70 \mathrm{MW} / \mathrm{cm}^{2}\right)$, respectively. For $1 \mathrm{sec}$ time constant at the lock-in amplifier, a relative signal of $10^{-7}$ for $\Delta I_{S} / I_{S}$ can be detected.

\section{Fig. 3 Imaging non-fluorescent chromoproteins and chromogenic reporter for gene} expression. Imaging distributions of cytoplasmic chromoproteins gtCP (a) and cjBlue (c) in live E. coli cells, respectively, by stimulated emission microscopy. b and $\mathbf{d}$ are the corresponding wide-field transmission images. Plasmids containing the genes encoded for gtCP and cjBLue are transformed into E. coli, respectively. Compared to gtCP, cjBlue 
is expressed less abundantly inside cells. e, Stimulated emission imaging of the oxidation product (4,4'-dichloro-5,5'-dibromoindigo) resulting from X-gal hydrolysis catalyzed by $\beta$-galactosidase, the protein encoded by lacZ reporter gene. Indigo accumulation in $E$. coli cells is detected without any lacZ induction. The signal is detected only when both the cells and X-gal are present. Unlike the homogeneous protein images in a \& $\mathbf{c}$, indigo product shows inhomogeneous localized distribution inside cells due to its insolubility, as shown in the magnified image $\mathbf{f}$. The direct transmission image $\mathbf{g}$ shows the morphology of the same region. $\mathrm{gtCP}$ and cjBlue absorb around $580 \mathrm{~nm}^{18}$ and $610 \mathrm{~nm}^{19}$, respectively; indigo product has a broad absorption around $600 \sim 650 \mathrm{~nm}$. In a \& c, $590 \mathrm{~nm}$ and $660 \mathrm{~nm}$ for excitation and stimulation, respectively; in e \& $\mathbf{f}, 600 \mathrm{~nm}$ and $680 \mathrm{~nm}$ for excitation and stimulation, respectively. The excitation and stimulation beams of 0.1 and $0.3 \mathrm{~mW}$, respectively, and $\sim 0.3 \mathrm{ps}$ of time delay are used for all the above images. All of the images were taken between $5 \mathrm{sec}$ and $45 \mathrm{sec}$, depending on the image size.

Fig. 4 Transdermal drug distribution in 3D and microvascular imaging. $a \& b$, Drug delivery of toluidine blue $\mathrm{O}$ (TBO), a drug used as photosensitizer in photodynamic therapy, in a freshly cut mouse ear skin the same area at two different depths, 3 and 25 $\mu \mathrm{m}$, respectively, $30 \mathrm{~min}$ after topical application of $10 \mu \mathrm{M}$ TBO/PBS solution. At the surface layer $(3 \mu \mathrm{m})$ of stratum corneum, (a) shows TBO is accumulated in the protein phase of the polygonal cells rather than in the lipid-rich intercellular space. At the layer of epidermis $(25 \mu \mathrm{m})$, (b) shows a rich TBO distribution following the subcellular cytoplasm of nucleated basal keratinocytes. These images support the 'hydrophilic path' as a main pathway for transdermal drug delivery of TBO. $600 \mathrm{~nm}$ and $680 \mathrm{~nm}$ for 
excitation and stimulation, respectively. The excitation and stimulation beams have 0.1 and $0.3 \mathrm{~mW}$, respectively, at the focus. $\sim 0.3 \mathrm{ps}$ time delay is used. The color table (cyan hot) shows the magnitude of $\Delta I_{S} / I_{S}$. c, Ex vivo imaging of microvasculature network of a mouse ear based on endogenous hemoglobin contrast. Stimulated emission image (red channel, maximum intensity projection) shows the blood vessel network surrounding sebaceous glands (green channel, simultaneously recorded by confocal reflectance). Shown by the zoomed-in image, individual red blood cells are lined up within a single capillary ( $\sim 5 \mu \mathrm{m}$ in diameter). In c, $830 \mathrm{~nm}(\sim 20 \mathrm{~mW})$ and $600 \mathrm{~nm}(\sim 3 \mathrm{~mW})$ are used for two-photon excitation of Soret band and one-photon stimulated emission of $Q$ band of hemoglobin, respectively. Pulse widths of both excitation and stimulation beams are about 0.2 ps with $\sim 0.2 \mathrm{ps}$ time delay between them.

\section{METHODS SUMMARY}

In the scheme depicted in Fig. 1, two fs optical parametric oscillators (OPO) (Coherent/APE) are synchronously pumped by a fs mode-locked $76 \mathrm{MHz}$ Ti:Sapphire laser (Coherent). Two independent frequency-doubled outputs from these two OPO signal waves, in the wavelength range of 560 to $700 \mathrm{~nm}$ with pulse widths around $200 \mathrm{fs}$, serve as the either excitation or stimulation pulse trains. A pulse compressor consisting of a pair of SF11 prisms is built to control the pulse width. Collinear excitation and stimulation beams are combined and focused with a high numerical aperture $(\mathrm{NA}=1.2)$ objective onto a common focal spot. The temporal delay between the synchronized excitation and stimulation inter-pulse is adjusted to between 0.2 and $0.3 \mathrm{ps}$. The intensity 
of the excitation beam is modulated by an acousto-optical modulator (Crystal technology) at $5 \mathrm{MHz}$. A condenser with a N.A. $=0.9$ is used to collect the forward propagating stimulation beam, which is spectrally filtered before detected by a photodiode. To acquire images with a laser scanning microscope (FV300, Olympus), we used a $100 \mu$ s time constant for a lock-in amplifier (SR844, Stanford Research) and pixel dwell time of 190 $\mu \mathrm{s}$.

(1) Pawley, J. B. (ed.) Handbook of Biological Confocal Microscopy. Springer, 3rd edition.

(2) Lakowicz, J. R. Principles of Fluorescence Spectroscopy (Plenum Press, New York, 1983).

(3) Turro, N.J. Modern Molecular Photochemistry. University Science Books (1991).

(4) Einstein, A. On the quantum theory of radiation. Phys Z 18, 121 (1917).

(5) Seigman, A. E. Laser. University Science Books (1986).

(6) Hamilton, C. E., Kinsey, J. L. \& Field, R. W. Stimulated emission pumping: new methods in spectroscopy and molecular dynamics," Ann. Rev. Phys. Chem. 37, 493-524 (1986).

(7) Hell, S. W. \& Wichmann, J. Breaking the diffraction resolution limit by stimulated emission: stimulated-emission-depletion fluorescence microscopy. Opt. Lett. 19, 780-782 (1994). 
(8) Dong, C. Y., So, P. T., French, T. \& Gratton, E. Fluorescence lifetime imaging by asynchronous pump-probe microscopy. Biophys. J. 69, 2234-2242 (1995).

(9) Cantor, C. R. \& Schimmel, P. R. Biophysical Chemistry. W. H. Freeman (1980).

(10) Moerner, W. E. \& Kador, L. Optical detection and spectroscopy of single molecules in a solid. Phys. Rev. Lett. 62, 2535-2538 (1989).

(11) Ye, J., Ma, L. S. \& Hall, J. L. Ultrasensitive detections in atomic and molecular physics: demonstration in molecular overtone spectroscopy. J. Opt. Soc. Am. B 15, 6-15 (1998).

(12) Freudiger, C. W. et al. Label-free biomedical imaging with high sensitivity by stimulated Raman scattering microscopy. Science 322, 1857-1861 (2008).

(13) Fu, D. et al. High-resolution in vivo imaging of blood vessels without labeling. Opt. Lett. 32, 2641-2643 (2007).

(14) Denk, W., Strickler, J. H. \& Webb, W. W. Two-photon laser scanning fluorescence microscopy. Science 248, 73-76 (1990).

(15) Evans, C. L. \& Xie, X. S. Coherent anti-Stokes Raman scattering microscopy: chemical imaging for biology and medicine. Annu. Rev. Anal. Chem. 1, 883-909 (2008).

(16) Rittweger, E., Rankin, B. R., Westphal, V. \& Hell, S. W. Fluorescence depletion mechanisms in super-resolving STED microscopy. Chem. Phys. Lett. 442, 483-487 (2007).

(17) Du, H. et al. PhotochemCAD: A computer-aided design and research tool in photochemistry. Photochem. Photobiol., 68, 141-142 (1998). 
(18) Gurskaya, N. G. et al. GFP-like chromoproteins as a source of far-red fluorescent proteins. FEBS Lett. 507 (1), 16-20 (2001).

(19) Chan, M. C. Y. et al. Structural characterization of a blue chromoprotein and its yellow mutant from the sea anemone Cnidopus japonicus J. Biol. Chem. 281, 3781337819 (2006).

(20) Zhang, J., Campbell, R. E., Ting, A. Y. \& Tsien, R. Y. Creating new fluorescent probes for cell biology. Nature Review Molecular Biology 3, 906-918 (2002).

(21) Miller, J. H. Experiments in Molecular Genetics (Cold Spring Harbor Laboratory, New York, 1972).

(22) Cai, L., Friedman, N. \& Xie, X. S. Stochastic protein expression in individual cells at the single molecule level," Nature 440, 358-362 (2006).

(23) Tremblay, J. F. et al. Photodynamic therapy with toluidine blue in Jurkat cells: cytotoxicity, subcellular localization and apoptosis induction. Photochem. Photobiol. Sci. 1, 852-856 (2002).

(24) Chelvanayagam, D. K. \& Beazley, L. D. Toluidine blue-O is a Nissl bright-field counterstain for lipophilic fluorescent tracers Di-ASP, DiI and DiO. J. Neurosci. Meth. 72, 49-55 (1997).

(25) McDonald, D. M. \& Choyke, P. L. Imaging of angiogenesis: from microscope to clinic. Nature Med. 9, 713-725 (2003).

(26) Grinvald A., Lieke, E., Frostig, R. D., Gilbert C. D. \& Wiesel T. N. Functional architecture of cortex revealed by optical imaging of intrinsic signals. Nature, 324, 361364 (1986). 
(27) Kleinfeld, D., Mitra, P. P., Helmchen, F. \& Denk, W. Fluctuations and stimulusinduced changes in blood flow observed in individual capillaries in layers 2 through 4 of rat neocortex. Proc. Natl. Acad. Sci. USA 95, 15741-15746 (1998).

(28) Clay, G. O., Schaffer, C. B. \& Kleinfeld, D. Large two-photon absorptivity of hemoglobin in the infrared range of 780-880 nm. J. Chem. Phys. 126, 025102 (2007). (29) Wang, W. et al. Femtosecond multicolor pump-probe spectroscopy of ferrous cytochrome c. J. Phys. Chem. B 104, 10789-10801 (2000).

Supplementary Information is linked to the online version of the paper at www.nature.com/nature.

Acknowledgements We thank K. Lukyanov and A. Miyawaki for providing the generous gifts of chromoprotein gtCP and cjBlue plasmid DNA, respectively; Coherent Inc. for providing a loan for one fs-OPO; P. Choi for preparing X-gal E. coli cells; and B. G. Saar, C. W. Freudiger, S. Basu, J. W. Lichtman and C. B. Schaffer for helpful discussions. This work was supported by National Science Foundation (grant CHE0634788) and U.S Department of Energy's Basic Energy Sciences Program (DE-FG0207ER15875). Harvard University has filed a patent application based on this work.

Author Contributions W. M., S. L. and S. C. performed experiments and analyzed data. R. R. constructed E. coli cells expressing chromoproteins. G. R. H. and S. C. helped to construct the laser systems. W. M., S. L. and X. S. X. conceived the concept, designed the experiments and wrote the paper. 
Author Information Reprints and permissions information is available at www.nature.com/reprints. Correspondence and requests for materials should be addressed to X. S. X.(xie@chemistry.harvard.edu). 
a

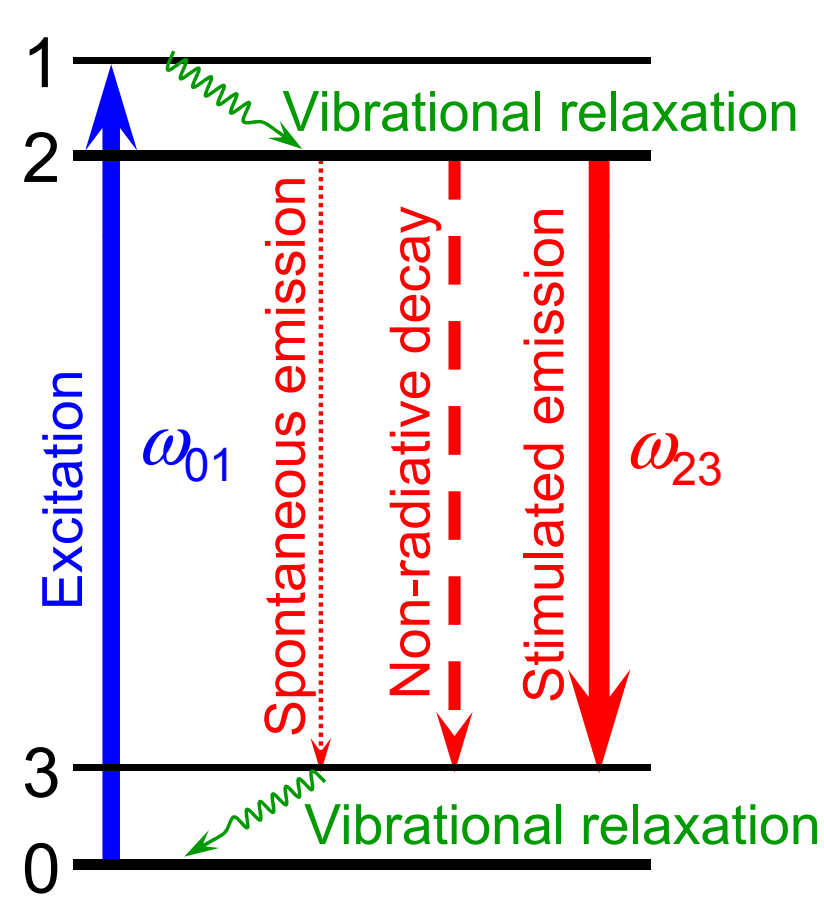

b

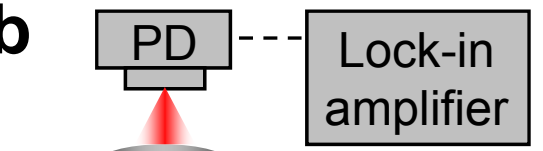

$\frac{\Delta I_{E}}{I_{E}}=-\frac{\sigma_{a b s .[0 \rightarrow 1]}}{S}$

$\frac{\Delta I_{S}}{I_{S}}=\frac{\sigma_{\text {sti.em }[2 \rightarrow 3]}}{S}$

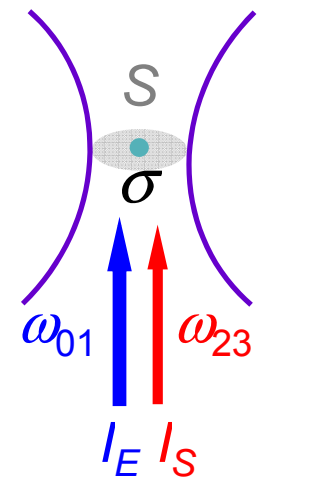

Stimulation beam

C

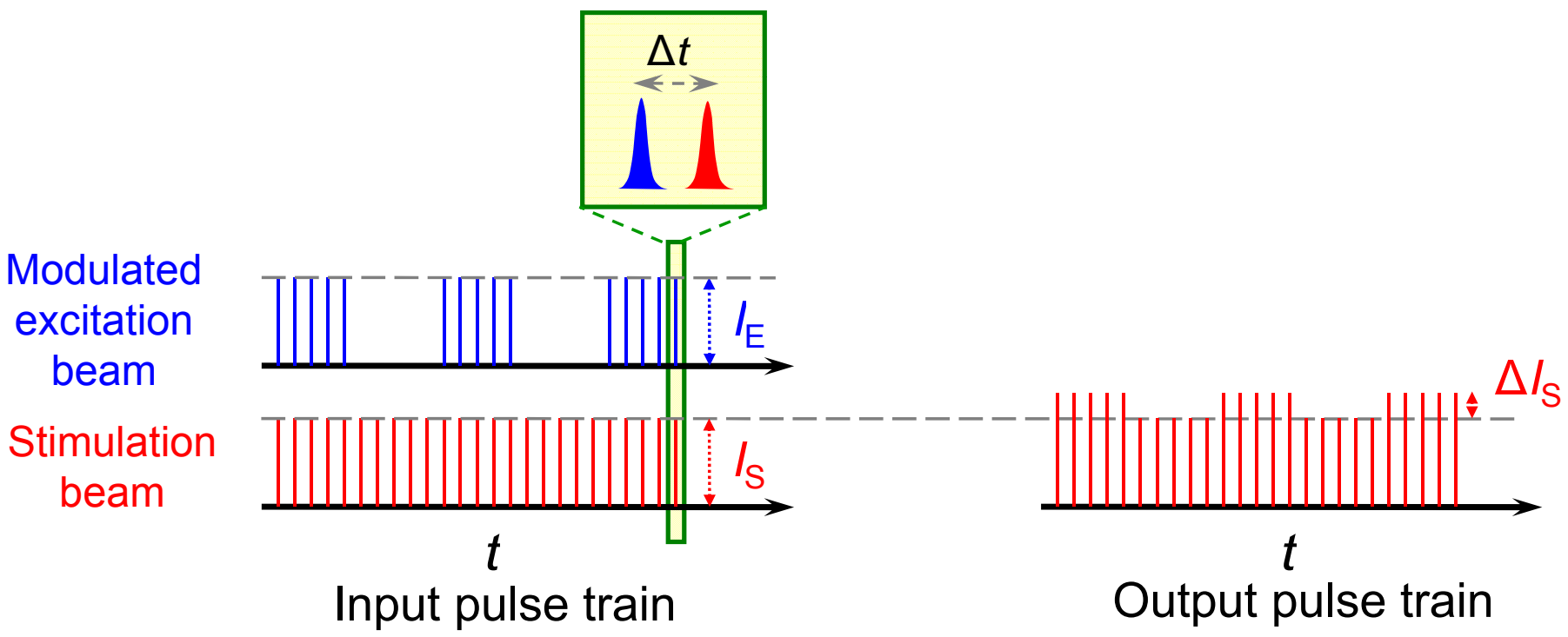

Figure 1 

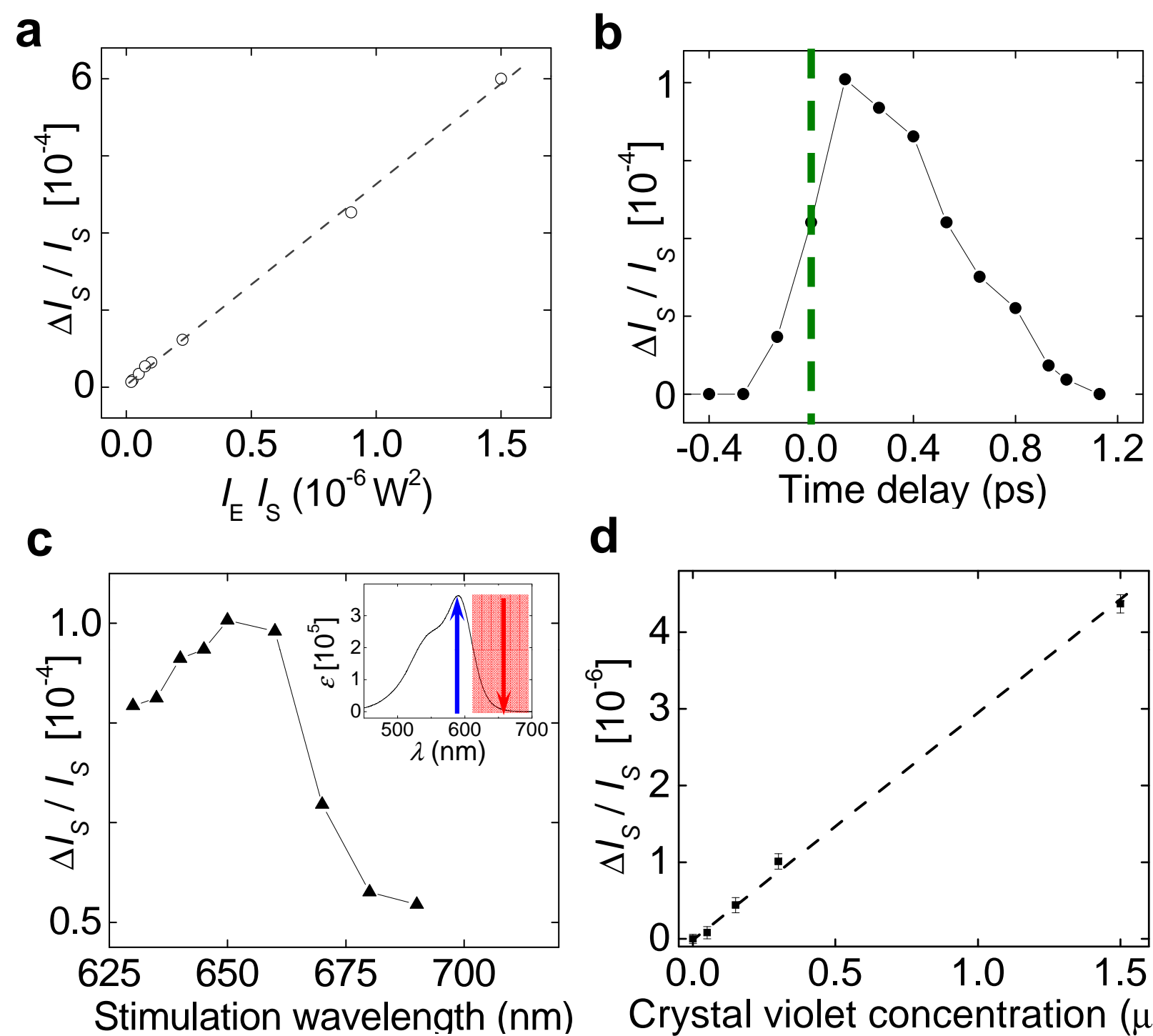

d

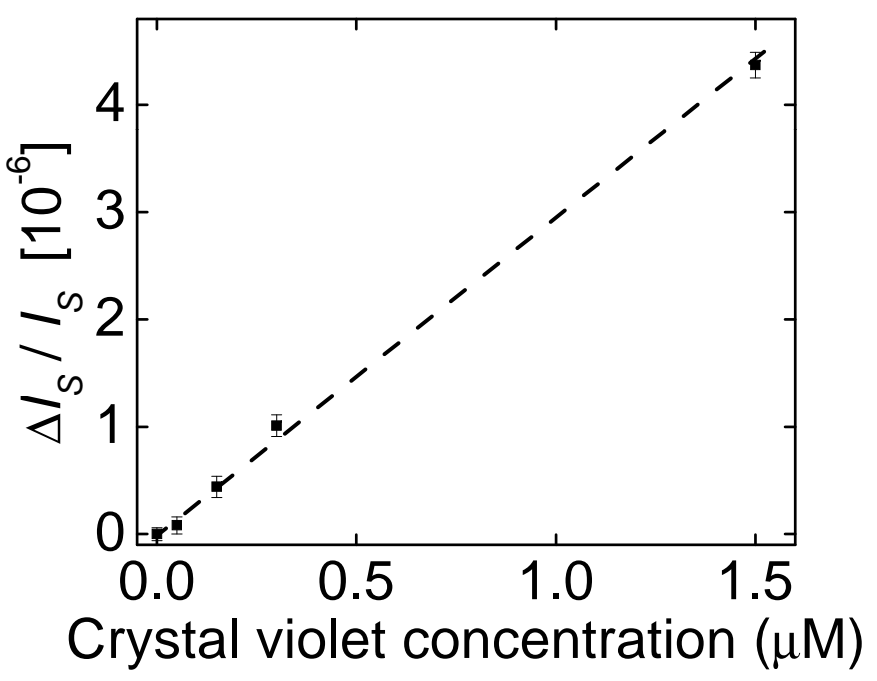

Figure 2 

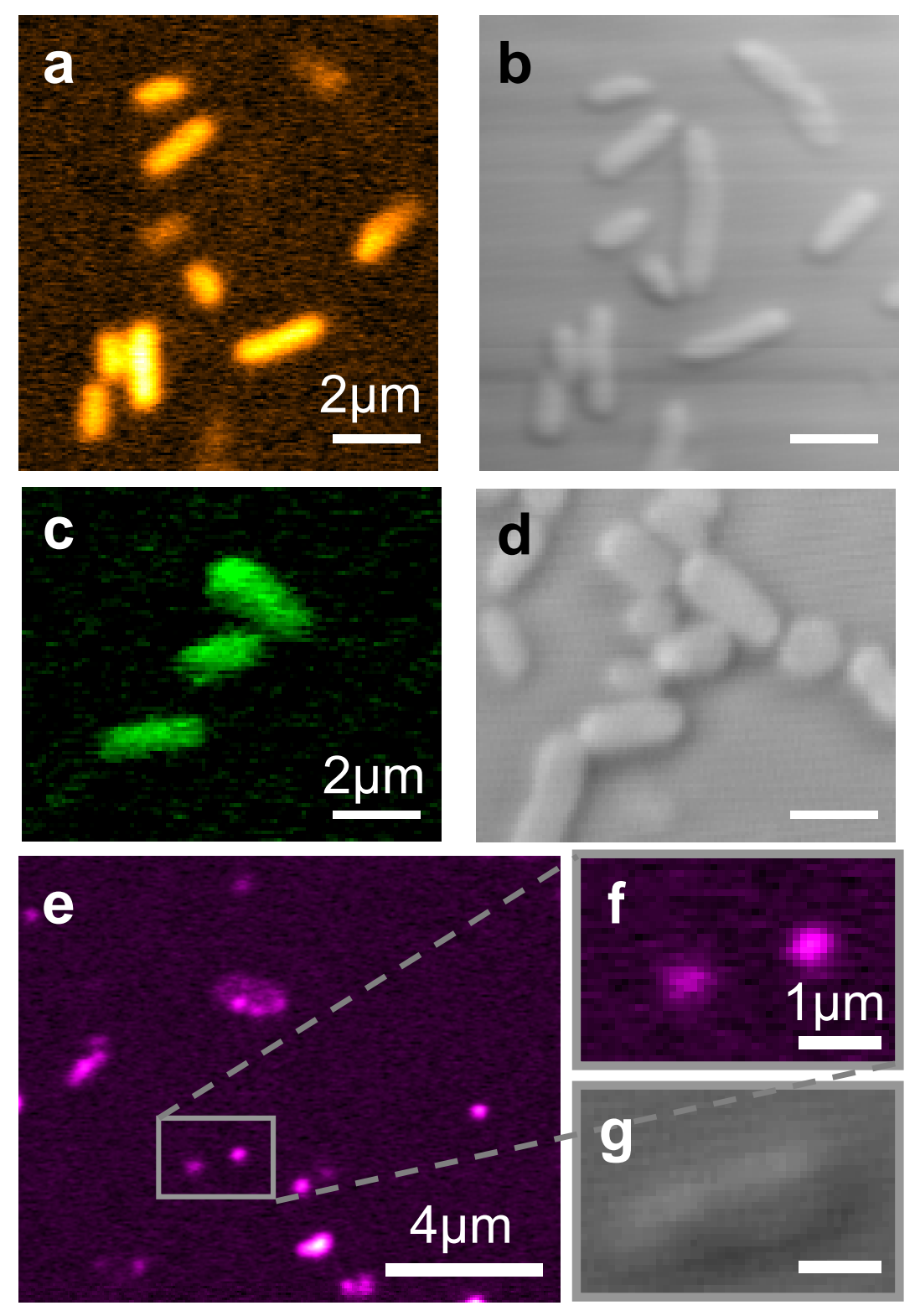

Figure 3 

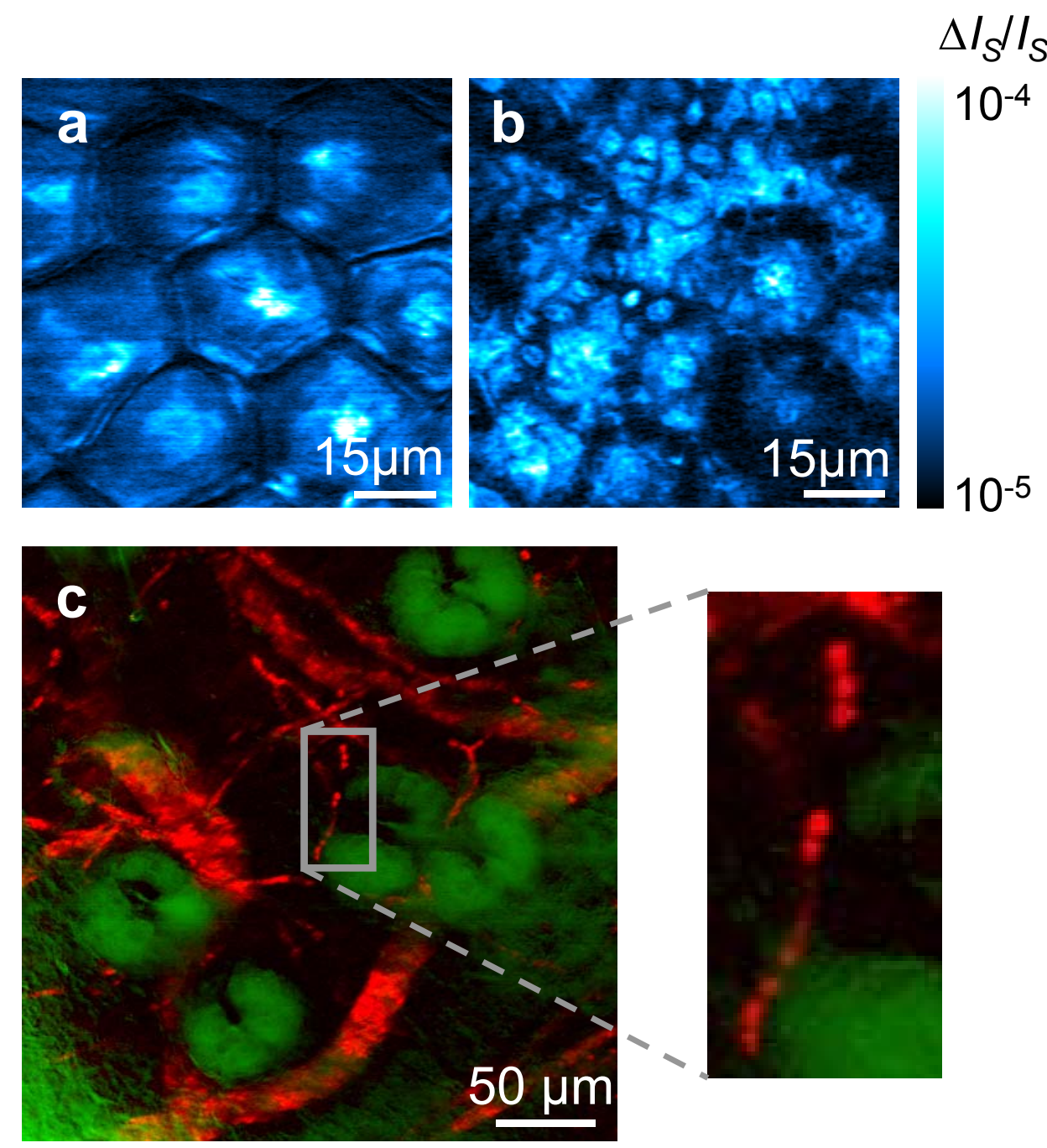

Figure 4 